\title{
Pressure enhanced thermoelectric properties in $\mathrm{Mg}_{2} \mathrm{Sn}$
}

\author{
San-Dong Guo and Jian-Li Wang \\ Department of Physics, School of Sciences, China University of Mining and Technology, Xuzhou 221116, Jiangsu, China
}

\begin{abstract}
Pressure dependence of electronic structures and thermoelectric properties of $\mathrm{Mg}_{2} \mathrm{Sn}$ are investigated by using a modified Becke and Johnson (mBJ) exchange potential, including spin-orbit coupling (SOC). The corresponding value of spin-orbit splitting at $\Gamma$ point is $0.47 \mathrm{eV}$, which is in good agreement with the experimental value $0.48 \mathrm{eV}$. With the pressure increasing, the energy band gap first increases, and then decreases. In certain doping range, the power factor for n-type has the same trend with energy band gap, when the pressure increases. Calculated results show that the pressure can lead to significantly enhanced power factor in n-type doping below the critical pressure, and the corresponding lattice thermal conductivity near the critical pressure shows the relatively small value. These results make us believe that thermoelectric properties of $\mathrm{Mg}_{2} \mathrm{Sn}$ can be improved in n-type doping by pressure.
\end{abstract}

PACS numbers: 72.15.Jf, 71.20.-b, 71.70.Ej, 79.10.-n

Keywords: Spin-orbit coupling; Pressure; Power factor, Lattice thermal conductivity

Thermoelectric material by using the Seebeck effect can convert waste heat directly to electricity to solve energy problems. The performance of thermoelectric material can be characterized by dimensionless figure of merit[1, 2], $Z T=S^{2} \sigma T /\left(\kappa_{e}+\kappa_{L}\right)$, where S, $\sigma$, $\mathrm{T}, \kappa_{e}$ and $\kappa_{L}$ are the Seebeck coefficient, electrical conductivity, absolute temperature, the electronic and lattice thermal conductivities, respectively. Bismuthtellurium systems $[3,4]$, silicon-germanium alloys $[5,6]$, lead chalcogenides $[7,8]$ and skutterudites $[9,10]$ have been identified as excellent thermoelectric material for thermoelectric devices. For thermoelectric research, the main objective is to search for high $Z T$ materials, which has proven to be interesting and challenging.

The thermoelectric material $\mathrm{Mg}_{2} \mathrm{X}(\mathrm{X}=\mathrm{Si}, \mathrm{Ge}, \mathrm{Sn})$ composed of abundant, low-cost elements and their alloys have attracted much recent attention[11-13], and various doping strategies have been adopted to attain high $Z T[14-16]$. Pressure by tuning the electronic structures of materials can accomplish many interesting phenomenons like recent pressure-induced high-Tc superconductivity in $\left(\mathrm{H}_{2} \mathrm{~S}\right)_{2} \mathrm{H}_{2}[17,18]$. Here, we use first-principle calculations and Boltzmann transport theory to address the pressure dependence of thermoelectric properties in the $\mathrm{Mg}_{2} \mathrm{Sn}$. Calculated results show that the pressure dependence of energy band gap with $\mathrm{mBJ}+\mathrm{SOC}$ is consistent with one with $\mathrm{mBJ}[19]$, and first increases, and then decreases. Pressure can significantly improve power factor in n-type doping below the critical pressure. It is found that pressure can reduce the lattice thermal conductivity in certain pressure range. These lead to enhanced $Z T$, and make $\mathrm{Mg}_{2} \mathrm{Sn}$ become more efficient for thermoelectric application in n-type doping by pressure. So, pressure tuning offers a very effective method to search for materials with enhanced thermoelectric properties.

The rest of the paper is organized as follows. Firstly, we shall give our computational details. Secondly, we
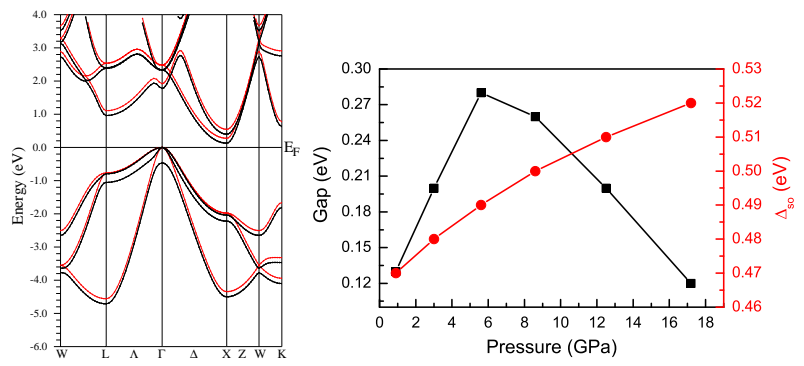

FIG. 1. (Color online) Left: The energy band structures by using $\mathrm{mBJ}$ (Red lines) and $\mathrm{mBJ}+\mathrm{SOC}$ (Black lines). Right: The energy band gap (Gap) and the value of spin-orbit splitting at $\Gamma$ point $\left(\Delta_{s o}\right)$ as a function of pressure by using $\mathrm{mBJ}+\mathrm{SOC}$.

shall present our main calculated results and analysis. Finally, we shall give our discussion and conclusion.

We use a full-potential linearized augmented-planewaves method within the density functional theory (DFT) [20], as implemented in the package WIEN2k [21]. We use Tran and Blaha's mBJ exchange potential plus local-density approximation (LDA) correlation potential for the exchange-correlation potential [22] to do our main DFT calculations. The full relativistic effects are calculated with the Dirac equations for core states, and the scalar relativistic approximation is used for valence states [23-25]. The SOC was included self-consistently by solving the radial Dirac equation for the core electrons and evaluated by the second-variation method[26]. We use $6000 \mathrm{k}$-points in the first Brillouin zone for the self-consistent calculation. We make harmonic expansion up to $l_{\max }=10$ in each of the atomic spheres, and set $\mathrm{R}_{\mathrm{mt}} * \mathrm{k}_{\max }=8$. The self-consistent calculations are considered to be converged when the integration of the absolute charge-density difference between the input and output electron density is less than $0.0001|e|$ per formula unit, where $e$ is the electron charge. Transport calcula- 

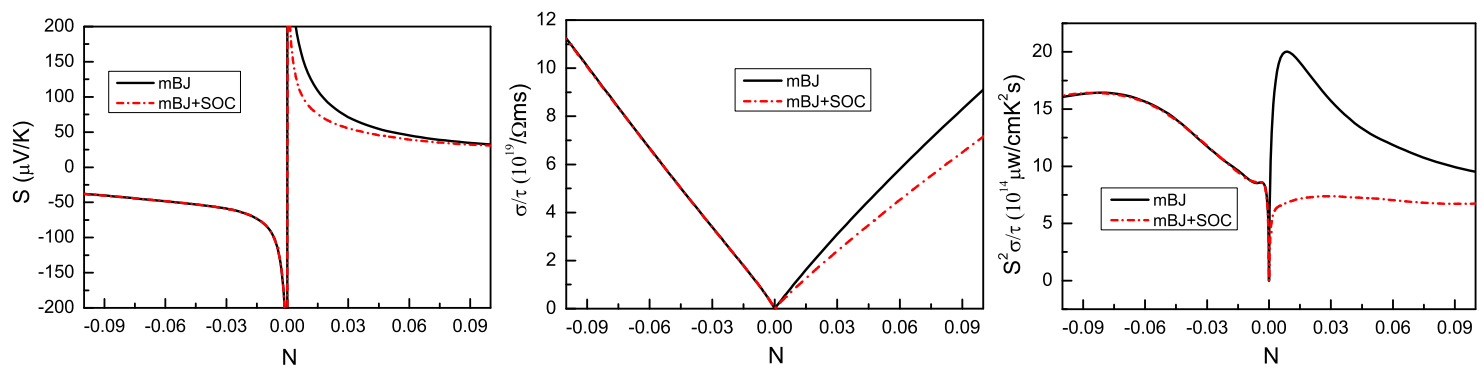

FIG. 2. (Color online) At temperature of $300 \mathrm{~K}$, transport coefficients as a function of doping levels (electrons [minus value] or holes [positive value] per unit cell): Seebeck coefficient S (Left), electrical conductivity with respect to scattering time $\sigma / \tau$ (Middle) and power factor with respect to scattering time $\mathrm{S}^{2} \sigma / \tau$ (Right) calculated with mBJ (Black solid line) and mBJ+SOC (Red dotted line).
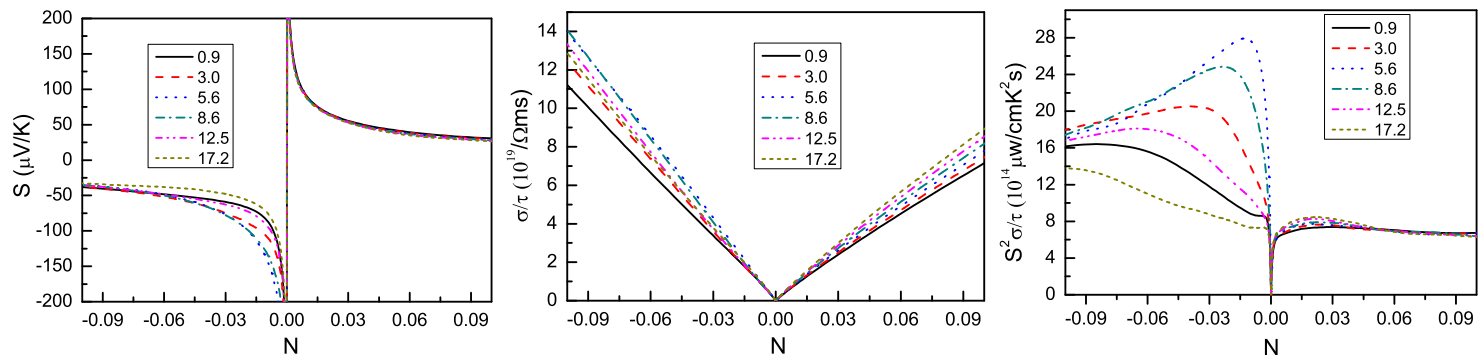

FIG. 3. At temperature of $300 \mathrm{~K}$, transport coefficients as a function of doping levels (electrons [minus value] or holes [positive value] per unit cell): Seebeck coefficient S (Left), electrical conductivity with respect to scattering time $\sigma / \tau$ (Middle) and power factor with respect to scattering time $\mathrm{S}^{2} \sigma / \tau$ (Right) with pressure being 0.9, 3.0, 5.6, 8.6, 12.5 and 17.2 (unit: GPa) calculated by using $\mathrm{mBJ}+\mathrm{SOC}$.

tions are performed through solving Boltzmann transport equations within the constant scattering time approximation as implemented in BoltzTrap[27], which has been applied successfully to several materials[28-30]. To obtain accurate transport coefficients, we use $200000 \mathrm{k}$-points in the first Brillouin zone for the energy band calculation. The lattice thermal conductivities are calculated by using Phono3py+VASP codes[31-34]. For the thirdorder force constants, $2 \times 2 \times 2$ supercells are built, and reciprocal spaces of the supercells are sampled by $2 \times 2 \times 2$ meshes. To compute lattice thermal conductivities, the reciprocal spaces of the primitive cells are sampled using the $13 \times 13 \times 13$ meshes.

The electronic structures, optical properties and thermoelectric properties of $\mathrm{Mg}_{2} \mathrm{Sn}$ at hydrostatic pressure have been investigated by mBJ exchange-potential[19]. However, SOC is very important for power factor calculations[35]. Here, we investigate the electronic structures and thermoelectric properties by using mBJ+SOC. First, the energy band structures of $\mathrm{Mg}_{2} \mathrm{Sn}$ with $\mathrm{mBJ}$ and $\mathrm{mBJ}+\mathrm{SOC}$ are shown in Figure 1. It is found that the SOC has little effect on the conduction bands, and has obvious influence on valence bands. The SOC splits the valence band at $\Gamma$ point, and the corresponding value of spin-orbit splitting $0.47 \mathrm{eV}$ is in good agreement with the experimental value $0.48 \mathrm{eV}[36]$. As expected, the SOC reduces the energy band gap due to the conduction band minimum (CBM) moving toward lower energy. The energy band gap and value of spinorbit splitting at $\Gamma$ point as a function of pressure by using $\mathrm{mBJ}+\mathrm{SOC}$ are present in Figure 1. The trend of energy band gap with $\mathrm{mBJ}+\mathrm{SOC}$ is consistent with one with $\mathrm{mBJ}[19]$, and first increases, and then decreases with increasing pressure. The explanation of trend of energy band gap can be found in ref.[19]. But, the spinorbit splitting monotonically increases.

$\mathrm{Mg}_{2} \mathrm{Sn}$ based thermoelectric materials are considered as potential candidates for efficient thermoelectricity. The pressure dependence of the semi-classic transport coefficients as a function of doping level is investigated within constant scattering time approximation Boltzmann theory. We firstly consider the SOC effects on the Seebeck coefficient S, electrical conductivity with respect to scattering time $\sigma / \tau$ and power factor with respect to scattering time $\mathrm{S}^{2} \sigma / \tau$, and they as a function of doping levels at the temperature of $300 \mathrm{~K}$ by using $\mathrm{mBJ}$ and $\mathrm{mBJ}+\mathrm{SOC}$ are present in Figure 2. It is clearly seen that the negative doping levels (n-type doping) show the negative Seebeck coefficient, and the positive doping levels (p-type doping) imply the positive Seebeck coefficient. Calculated results show that SOC has a detrimental influence on $\mathrm{S}, \sigma / \tau$ and $\mathrm{S}^{2} \sigma / \tau$ in p-type doping, but has a 


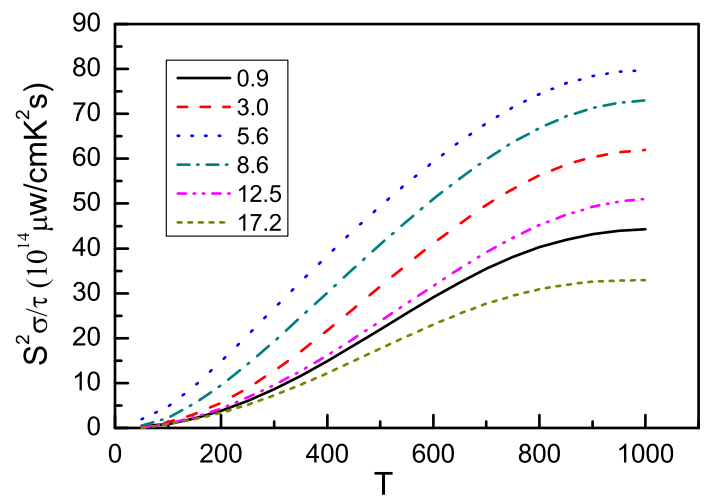

FIG. 4. (Color online) Power factor with respect to scattering time $S^{2} \sigma / \tau$ as a function of temperature for n-type with pressure being $0.9,3.0,5.6,8.6,12.5$ and 17.2 (unit: GPa) calculated by using $\mathrm{mBJ}+\mathrm{SOC}$ with the doping concentration of $1 \times 10^{20} \mathrm{~cm}^{-3}$.

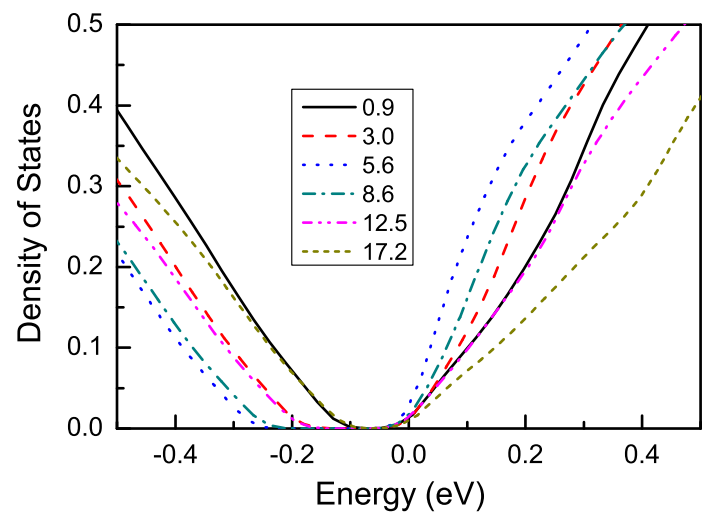

FIG. 5. (Color online) The total density of states with pressure being $0.9,3.0,5.6,8.6,12.5$ and 17.2 (unit: GPa) calculated by using $\mathrm{mBJ}+\mathrm{SOC}$. The Fermi level is defined at the conduction band minimum (CBM).

negligible effect in n-type doping. These can be explained by that SOC has larger influences on the valence bands than on the conduction bands. When SOC is absent, the best $\mathrm{p}$-type power factor is larger than the best n-type one. However, including SOC, the power factor in n-type doping is larger than one in $\mathrm{p}$ type doping in considered doping range, which agrees with the experimental results reporting high $Z T$ values for n-type than for p-type[14].

The pressure dependence of $\mathrm{S}, \sigma / \tau$ and $\mathrm{S}^{2} \sigma / \tau$ with pressure being $0.9,3.0,5.6,8.6,12.5$ and 17.2 (unit: GPa) calculated by using mBJ+SOC at temperature of $300 \mathrm{~K}$ are shown in Figure 3. It is interesting that $\mathrm{S}$ (absolute value), $\sigma / \tau$ and $\mathbf{S}^{2} \sigma / \tau$ have the same trend with energy band gap in certain doping range for n-type with the increasing pressure. When the pressure reaches the critical value of energy band gap, the $\mathrm{S}, \sigma / \tau$ and $\mathrm{S}^{2} \sigma / \tau$ attain the corresponding extremum. For p-type, the $\sigma / \tau$ has obvious dependence on pressure, but the $\mathrm{S}$ has very weak dependence on pressure, which leads to weak pressure

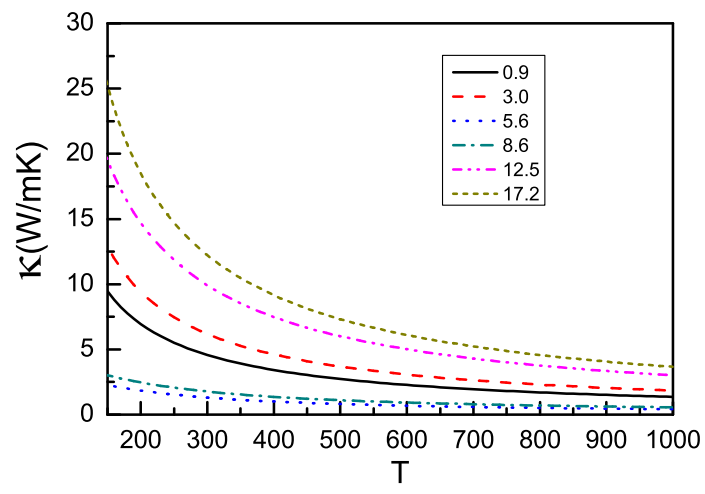

FIG. 6. (Color online) The lattice thermal conductivities $\kappa$ as a function of temperature with pressure being 0.9, 3.0, 5.6, 8.6, 12.5 and 17.2 (unit: GPa) calculated by using GGA.

dependence for $\mathrm{S}^{2} \sigma / \tau$. The strong pressure dependence for $\mathrm{S}^{2} \sigma / \tau$ in n-type doping shows that the $\mathrm{Mg}_{2} \mathrm{Sn}$ under pressure may become more efficient thermoelectric material. To clearly see interesting pressure dependence in n-type doping, the $S^{2} \sigma / \tau$ as a function of temperature with the doping concentration of $1 \times 10^{20} \mathrm{~cm}^{-3}$ are displayed in Figure 4. In the considered temperature range, the power factor always has the same trend with energy band gap.

To explain interesting pressure dependence of power factor in n-type doping, the total density of states with pressure being $0.9,3.0,5.6,8.6,12.5$ and 17.2 (unit: $\mathrm{GPa}$ ) calculated by using $\mathrm{mBJ}+\mathrm{SOC}$ are displayed in Figure 5. Calculated results imply that the slope of density of states of the conduction bands near the energy band gap first increases with the pressure increasing, and then decreases. The critical pressure happens to be the critical one for power factor. The large slope of density of states near the energy gap may induce a large Seebeck coefficient in narrow-gap semiconductors[37], leading to large power factor, which gives rise to the corresponding pressure dependence of power factor in n-type doping.

Finally, the lattice thermal conductivities $\kappa$ as a function of temperature with pressure being $0.9,3.0,5.6,8.6$, 12.5 and 17.2 calculated by using GGA are shown in Figure 6 . The lattice thermal conductivity is generally considered to be independent of doping, and typically goes as $1 / \mathrm{T}$. At high $\mathrm{T}$, it can reach the so-called minimum thermal conductivity. Calculated results show that the $\kappa$ near the critical pressure of energy band gap has the relatively small value, and the corresponding power factor in n-type doping has the relatively large one. These results imply that pressure can induce larger $Z T$ by reducing lattice thermal conductivity and enhancing power factor for n-type.

It has been proved that mBJ gap value agrees well with experimental value $0.3 \mathrm{eV}[19]$, but $\mathrm{mBJ}+\mathrm{SOC}$ gap value is less than experimental one. However, the $\mathrm{mBJ}+\mathrm{SOC}$ is more satisfactory than usual GGA or LDA+SOC in 
calculating electronic structure of $\mathrm{Mg}_{2} \mathrm{Sn}$. It is very important for power factor calculations to consider SOC for $\mathrm{MgSn}$, especially for p-type doping. When SOC is included, the n-type doping has more excellent power factor than p-type doping, which agrees that the best ptype material reported so far has lower $Z T$ than the best n-type. Pressure has obvious effects on the conduction bands, and has little influences on valence bands, which leads to remarkable effects on power factor for n-type and small effects in p-type doping.

In summary, we investigate pressure dependence of thermoelectric properties of $\mathrm{Mg}_{2} \mathrm{Sn}$ by using $\mathrm{mBJ}+\mathrm{SOC}$ . It is found that pressure can realize enhanced power factor below critical pressure and reduced lattice thermal conductivity near the critical pressure, which leads to the improved $Z T$ for efficient thermoelectric application. By choosing the appropriate doping concentration, $\mathrm{Mg}_{2} \mathrm{Sn}$ under pressure can provide great opportunities for efficient thermoelectricity.

This work is supported supported by the Fundamental Research Funds for the Central Universities (2015XKMS073). We are grateful to the Advanced Analysis and Computation Center of CUMT for the award of CPU hours to accomplish this work.

[1] Y. Pei, X. Shi, A. LaLonde, H. Wang, L. Chen and G. J. Snyder, Nature 473, 66 (2011).

[2] A. D. LaLonde, Y. Pei, H. Wang and G. J. Snyder, Mater. Today 14, 526 (2011).

[3] W. S. Liu, Q. Y. Zhang, Y. C. Lan, S. Chen, X. Yan, Q. Zhang, H. Wang, D. Z. Wang, G. Chen and Z. F. Ren, Adv. Energy Mater. 1, 577 (2011).

[4] D. K. Ko, Y. J. Kang and C. B. Murray, Nano Lett., 11, 2841 (2011).

[5] M. Zebarjadi, et al. Nano Lett. 11, 2225 (2011).

[6] B. Yu, et al. Nano Lett. 12, 2077 (2012).

[7] Y. Z. Pei, X. Y. Shi, A. Lalonde et al, Nature 473, 66 (2011).

[8] J. Q. He, J. R. Sootsman, S. N. Girard et al, J. Am. Chem. Soc. 132, 8669 (2010).

[9] A. C. Sklad, M. W. Gaultois and A. P. Grosvenor, J. Alloys Compd. 505, L6 (2010).

[10] X. Shi, J. Yang and J. R. Salvador, J. Am. Chem. Soc. 133, 7837 (2011).

[11] W. Liu, X. Tan, K. Yin, H. Liu, X. Tang, J. Shi, Q. Zhang, and C. Uher, Phys. Rev. Lett. 108, 166601 (2012).

[12] W. J. Luo, M. J. Yang, Q. Shen, H. Y. Jiang and L.
Zhang, Adv. Mater. Res. 66, 33 (2009).

[13] M. Yang, W. Luo, Q. Shen, H. Jiang and L. Zhang, Adv. Mater. Res. 66, 17 (2009).

[14] V. K. Zaitsev, M. I. Fedorov, E. A. Gurieva, I. S. Eremin, P. P. Konstantinov, A. Y. Samunin and M. V. Vedernikov, Phys. Rev. B 74, 045207 (2006).

[15] Q. Zhang, J. He, T. J. Zhu, S. N. Zhang, X. B. Zhao and T. M. Tritt, Appl. Phys. Lett. 93, 102109 (2008).

[16] W. Liu, X. Tang and J. Sharp, J. Phys. D: Appl. Phys. 43, 085406 (2010).

[17] D. F. Duan, Y. X. Liu, F. B. Tian, D. Li, X. L. Huang, Z. L. Zhao, H. Y. Yu, B. B. Liu, W. J. Tian and T. Cui, Sci. Rep. 4, 6968 (2014).

[18] A. P. Drozdov, M. I. Eremets, I. A. Troyan, V. Ksenofontov and S. I. Shylin, Nature 525, 73 (2015).

[19] S. D. Guo, EPL 109, 57002 (2015).

[20] P. Hohenberg and W. Kohn, Phys. Rev. 136, B864 (1964); W. Kohn and L. J. Sham, Phys. Rev. 140, A1133 (1965).

[21] P. Blaha, K. Schwarz, G. K. H. Madsen, D. Kvasnicka and J. Luitz, WIEN2k, an Augmented Plane Wave + Local Orbitals Program for Calculating Crystal Properties (Karlheinz Schwarz Technische Universität Wien, Austria) 2001, ISBN 3-9501031-1-2

[22] F. Tran and P. Blaha, Phys. Rev. Lett. 102, 226401 (2009).

[23] A. H. MacDonald, W. E. Pickett and D. D. Koelling, J. Phys. C 13, 2675 (1980).

[24] D. J. Singh and L. Nordstrom, Plane Waves, Pseudopotentials and the LAPW Method, 2nd Edition (Springer, New York, 2006).

[25] J. Kunes, P. Novak, R. Schmid, P. Blaha and K. Schwarz, Phys. Rev. B 64, 153102 (2001).

[26] D. D. Koelling, B. N. Harmon, J. Phys. C Solid State Phys. 10, 3107 (1977).

[27] G. K. H. Madsen and D. J. Singh, Comput. Phys. Commun. 175, 67 (2006).

[28] B. L. Huang and M. Kaviany, Phys. Rev. B 77, 125209 (2008).

[29] L. Q. Xu, Y. P. Zheng and J. C. Zheng, Phys. Rev. B 82, 195102 (2010).

[30] J. J. Pulikkotil, D. J. Singh, S. Auluck, M. Saravanan, D. K. Misra, A. Dhar and R. C. Budhani, Phys. Rev. B 86, 155204 (2012).

[31] G. Kresse, J. Non-Cryst. Solids 193, 222 (1995).

[32] G. Kresse and J. Furthmuller, Comput. Mater. Sci. 6, 15 (1996).

[33] G. Kresse and D. Joubert, Phys. Rev. B 59, 1758 (1999).

[34] A. Togo, L. Chaput and I. Tanaka, Phys. Rev. B 91, 094306 (2015).

[35] K. Kutorasinski, B. Wiendlocha, J. Tobola and S. Kaprzyk, Phys. Rev. B 89, 115205 (2014).

[36] F. Vazquez, A. R. Forman and M. Cardonna, Phys. Rev. 176, 905 (1968).

[37] M. ONOUE, F. ISHII and T. OGUCHI, J. Phys. Soc. Jpn. 77, 054706 (2008). 